\title{
Verallgemeinerung einiger Theoreme des Herrn Aronhold.
}

(Von Herrn S. Gudelfinger in Tübingen.)

In seiner ausgezeichneten Abhandlung: "Theorie der homogenen Functionen dritten Grades von drei Veränderlichen" (dieses Journal Bd. 55) berechnet Herr Aronhold das Formensystem für die zusammengesetzte Function $a f+b \Delta f^{*}$ ) namentlich vermittelst der wichtigen Gleichungen (8.) und (29.) in \$. 28 daselbst. Die in diesen beiden Gleichungen ausgedrückten Sätze lassen sich nach mehreren Richtungen hin erweitern, was hier näher ausgeführt werden soll.

I. Die eben erwähnte Formel (8.) in $\$$. 28 oder, was dasselbe, das $25^{\text {te }}$ Theorem der Abhandlung lässt sich so fassen:

Bedeutet $\varphi$ irgend eine Form von $f$, von der Ordnung $\gamma$ in den Coefficienten, und setzt man

(1.) $\varphi_{a f+b \Delta f}=a^{\gamma} \varphi+a^{\gamma-1} b \varphi^{\prime}+\frac{1}{1.2} a^{\gamma-2} b^{2} \varphi^{\prime \prime}+\cdots+\frac{1}{1.2 .3 \ldots k} a^{\gamma-k} b^{\gamma} \varphi^{(k)}+\cdots$,

so ist

(2.) $\quad(\delta \varphi)_{a f+b \Delta f}=\varphi_{a f+b \Delta f}^{\prime}=\frac{1}{4}\left\{\frac{\partial G}{\partial a} \frac{\partial \varphi_{a f+b \Delta f}}{\partial b}-\frac{\partial G}{\partial b} \frac{\partial \varphi_{a f+b \Delta f}}{\partial a}\right\}$.

Dieser Satz ist nur ein specieller Fall der folgenden Formel:

$$
\left\{\begin{array}{c}
\boldsymbol{4}^{k} \varphi_{a f+b \Delta f}^{(k)}=\left(\frac{\partial G}{\partial a}\right)^{k} \frac{\partial^{k} \varphi_{a f+b \Delta f}}{\partial b^{k}}-k\left(\frac{\partial G}{\partial a}\right)^{k-1} \frac{\partial G}{\partial b} \frac{\partial^{k} \varphi_{a f+b \Delta f}}{\partial b^{k-1} \partial a} \\
+\frac{k(k-1)}{2}\left(\frac{\partial G}{\partial a}\right)^{k-2}\left(\frac{\partial G}{\partial b}\right)^{2} \frac{\partial^{k} \varphi_{a f+b \Delta f}}{\partial b^{k-2} \partial a^{2}}+\cdots+(-1)^{k}\left(\frac{\partial G}{\partial b}\right)^{k} \frac{\partial^{k} \varphi_{a f+b \Delta f}}{\partial a^{k}}
\end{array}\right.
$$

Der Beweis hierfür ist genau derselbe, wie der von Aronhold für die Gleichung (2.) aufgestellte; man hat dabei nur noch Regeln aus der Differentialrechnung anzuwenden, die auch zur Ableitung des Taylorschen Lehrsatzes für mehrere Veränderliche gebraucht werden.

*) Wir werden, wenn nicht ausdrücklich das Gegentheil bemerkt ist, uns stets der Bezeichnungsweise des Herrn Aronhold bedienen und auf dessen Arbeit einfach mit dem Worte "Abhandlung" verweisen. 
II. Die weiteren Erweiterungen der Aronholdschen Formeln beziehen sich auf das Formensystem für die Grundfunction $a P_{f}-b R_{f}$. Gerade wie nämlich die Gleichungen (8.) und (29.) in $\$$. 28 der Abhandlung die meisten Formenbildungen für die zusammengesetzte Grundfunction $a f+b \Delta f$ liefern, so existiren analoge Theoreme, die das gleiche leisten, wenn $a P_{f}-b R_{f}$ als Grundform angenommen wird.

Um zu denselben zu kommen, gehen wir von der Beziehung aus:

$$
\text { (4.) } \left.\quad \Delta\left(a P_{f}-b R_{f}\right)=-\frac{1}{2} R\left\{\frac{\partial S_{a b}}{\partial b} P_{f}+\frac{\partial S_{a b}}{\partial a} R_{f}\right\}^{*}\right) \text {. }
$$

Haben nun $\varphi, \varphi^{\prime}, \varphi^{\prime \prime}$ etc. wieder dieselbe Bedeutung wie in (1.), so erhält man z. B. $\varphi_{a P_{f}-b R_{f}}^{\prime}$, wenn man in **)

$$
\varphi^{\prime}=\Sigma ! \frac{\partial \varphi}{\partial a_{x \lambda \mu}} b_{x \lambda \mu}
$$

$a_{x \lambda \mu}$ durch $a p_{x \lambda \mu}-b r_{x \lambda \mu}$ und also nach (4.)

ersetzt; es wird somit

$$
b_{x \lambda \mu} \quad \text { durch }-\frac{\boldsymbol{R}}{2}\left\{\frac{\partial \boldsymbol{S}_{a b}}{\partial \boldsymbol{b}} p_{x \lambda \mu}+\frac{\partial \boldsymbol{S}_{a b}}{\partial \boldsymbol{a}} \boldsymbol{r}_{z \lambda \mu}\right\}
$$

$$
\boldsymbol{\varphi}_{a P_{f}-b R_{f}}^{\prime}=-\frac{\boldsymbol{R}}{2} \Sigma ! \frac{\partial \varphi_{a P_{f}-b R_{f}}}{\partial\left(a p_{x \lambda \mu}-b r_{x \lambda \mu}\right)}\left(\frac{\partial S_{a b}}{\partial b} p_{x \lambda \mu}+\frac{\partial S_{a b}}{\partial a} r_{x \lambda \mu}\right)
$$

oder

$$
\text { (5.) } \quad \varphi_{a P_{f}-b R_{f}}^{\prime}=\frac{\boldsymbol{R}}{2}\left\{\frac{\partial \boldsymbol{S}_{a b}}{\partial a} \frac{\partial \boldsymbol{\varphi}_{a} \boldsymbol{P}_{f}-b R_{f}}{\partial b}-\frac{\partial \boldsymbol{S}_{a b}}{\partial b} \frac{\partial \boldsymbol{\varphi}_{a \boldsymbol{P}_{f}-b R_{f}}}{\partial \boldsymbol{a}}\right\} \text {. }
$$

In ganz derselben Weise findet man allgemein:

$$
\left\{\begin{array}{l}
\boldsymbol{\varphi}_{a P_{f}-b \boldsymbol{R}_{f}}^{(k)}=(-1)^{k} \frac{\boldsymbol{R}^{k}}{2^{k}}\left\{\frac{\partial^{k} \boldsymbol{\varphi}_{a \boldsymbol{P}_{f}-b \boldsymbol{R}_{f}}}{(\partial a)^{k}}\left(\frac{\partial \boldsymbol{S}_{a b}}{\partial b}\right)^{k}-k \frac{\partial^{k} \boldsymbol{\varphi}_{a} \boldsymbol{P}_{f}-b \boldsymbol{R}_{f}}{\partial a^{k-1} \partial b}\left(\frac{\partial \boldsymbol{S}_{a b}}{\partial b}\right)^{k-1} \frac{\partial \boldsymbol{S}_{a b}}{\partial a}\right. \\
\left.+\frac{k(k-1)}{2} \frac{\partial^{k} \boldsymbol{\varphi}_{a \boldsymbol{P}_{f}-b \boldsymbol{R}_{f}}}{\partial \boldsymbol{a}^{k-2} \partial \boldsymbol{b}^{2}}\left(\frac{\partial \boldsymbol{S}_{a b}}{\partial \boldsymbol{b}}\right)^{k-2}\left(\frac{\partial \boldsymbol{S}_{a b}}{\partial a}\right)^{2}-\cdots+(-1)^{k} \frac{\partial^{k} \boldsymbol{\varphi}_{a} \boldsymbol{P}_{f}-b \boldsymbol{R}_{f}}{\partial \boldsymbol{b}^{k}}\left(\frac{\partial \boldsymbol{S}_{a b}}{\partial a}\right)^{k}\right\}
\end{array}\right.
$$

Aus (5.) lässt sich über Combinanten ein interessanter Satz ableiten. Ist nämlich $\varphi$ eine Combinante von $f$ und $\Delta f$, so wird $\varphi^{\prime}$ und somit auch $\varphi_{a P_{f}-b R_{f}}^{\prime}$ identisch Null, und die Gleichung (5.) geht dann in diese über:

$$
\mathbf{0}=\frac{\partial \boldsymbol{S}_{a b}}{\partial a} \frac{\partial \varphi_{a P_{f}-b R_{f}}}{\partial b}-\frac{\partial \boldsymbol{S}_{a b}}{\partial b} \frac{\partial \varphi_{a P_{f}-b R_{f}}}{\partial a} .
$$

*) Auf diese Form lässt sich leicht die Gleichung IV. auf Seite 191 der Abhandlung bringen. Für den Beweis derselben und überhaupt der damit zusammenhängenden Formeln vergleiche man \$. 7 meiner Inauguralschrift: Zur Theorie des simultanen Systems einer cubischen und einer biquadratischen binären Form.

**) Das Zeichen $\Sigma$ ! brauche ich in dem Aronholdschen Sinne (Bd. 55 S. 129 dieses Journals), dass nämlich die Summation nicht auf $x, \lambda, \mu$ einzeln ausgedehnt wird, sondern jede Combination $x, \lambda, \mu$ in der Summe nur einfach zu nehmen ist. 
Nach einem bekannten Theoreme Jacobis über Functionaldeterminanten ist daher $\varphi_{a p_{f}-b R_{f}}$ eine Function und zwar - da $\varphi$ ganz und homogen in $a$ und $b$ - eine gewisse Potenz von $S_{a b}$, so dass man setzen kann:

$$
\text { (7.) } \quad \varphi_{a P_{f}-b R_{f}}=A . S_{a b}^{\nu} \text {. }
$$

In dieser Gleichung bedeutet $A$ eine von $a$ und $b$ unabhängige Form, die, wie sich leicht beweisen lässt, eine Combinante von $f$ und $\Delta f$ sein muss.

In der That macht man in (7.) $a=1$ und $b=0$, so folgt zunächst

$$
\varphi_{p_{f}}=A . S^{\nu}
$$

ersetzt man hierin $f$ durch $a f+b \Delta f$ und vergleicht die hieraus resultirende Gleichung mit (7.), so erhält man

$$
A_{n f+b \Delta f}=A \cdot G^{8 \nu},
$$

welche Beziehung die bekannte Bedingung dafür ausdrückt, dass $A$ eine Combinante von $f$ und $\Delta f$ ist.

III. Die in I. und II. aufgestellten Relationen sind alle als Verallgemeinerungen des $25^{\text {ten }}$ Theorems der Aronholdschen Abhandlung zu betrachten. Eine Ausdehnung der Gleichung (29.) in $\$$. 28 daselbst findet ihren Ausdruck in folgendem Satze:

Bezeichnet man eine beliebige Invariante $\chi$, gebildet für $a P_{f}-b R_{f}$ als Grundform, durch $\chi_{a p-b r}$ und setzt

$$
\chi_{a P_{f}-b R_{f}}=\Sigma ! \frac{\partial \chi_{a p-b r}}{\partial\left(a p_{x \lambda \mu}-b r_{x \lambda \mu}\right)} x_{x} x_{\lambda} x_{\mu},
$$

so findet die Beziehung statt:

$$
\text { (8.) } \quad 6 R \chi_{a P_{f}-b R_{f}}=\frac{\partial \chi_{a p-b r}}{\partial a} \Delta f-\frac{\partial \chi_{a p-b r}}{\partial b} f \text {. }
$$

Der Beweis ist sehr einfach. $\chi_{p_{f}}$ ist als eine Covariante dritten Grades eine lineare Combination von $f$ und $\Delta f$ (Abhandlung Seite 184), so dass man annehmen kann:

$$
\Sigma \frac{\partial \chi_{p}}{\partial p_{x \lambda \mu}} x_{x} x_{\lambda} x_{\mu}=A f+B \Delta f \text {. }
$$

Substituiren wir in dieser Gleichung für $x_{x} x_{\lambda} x_{\mu}$ ein Mal $p_{x \lambda \mu}$ und ein ander Mal $r_{x \lambda \mu}$, so erhalten wir, wenn $\gamma$ die Ordnung der Coefficienten von $f$ in $\chi$ bedeutet (Abh. $\$ .27,4$ und 16):

und wir haben demnach

$$
6 R . B=\gamma \chi_{p}, \quad 6 R \cdot A=\Sigma ! \frac{\partial \chi_{p}}{\partial p_{x \lambda \mu}} r_{x \lambda \mu},
$$


178 Gundelfinger, Verallgemeinerung einiger Theoreme des Herrn Aronhold.

da

(9.) $\quad\left\{\begin{aligned} 6 R \Sigma ! \frac{\partial \chi_{p}}{\partial p_{x \lambda \mu}} x_{x} x_{\lambda} x_{\mu} & =\gamma \chi_{p} \Delta f+\left(\Sigma ! \frac{\partial \chi_{p}}{\partial p_{x \lambda \mu}} r_{x \lambda \mu}\right) f, \\ & =\gamma \chi_{p} \Delta f-\delta\left(\chi_{p}\right) f,\end{aligned}\right.$

$$
\Sigma ! \frac{\partial \chi_{p}}{\partial p_{x \lambda \mu}} r_{x \lambda \mu}=-\sum_{\rho, \sigma, \tau} !_{x, \lambda, \mu} ! \frac{\partial \chi_{p}}{\partial p_{x \lambda \mu}} \frac{\partial p_{x \lambda \mu}}{\partial a_{\rho \sigma \tau}} b_{\rho \sigma \tau}=-\sum_{\rho, \sigma, \tau} ! \frac{\partial \chi_{p}}{\partial a_{\rho \sigma \tau}} b_{\rho \sigma \tau}=-\delta\left(\chi_{p}\right) .
$$

Ersetzt man jetzt in (9:) $f$ durch $a f+b \Delta f$, also $R$ durch $R G^{3}, \chi_{p}$ durch $G^{2 \gamma} \chi_{a p-b r}$ und nach (2.)

$$
\delta\left(\chi_{p}\right) \quad \text { durch } \cdot \frac{G^{2} \gamma}{4}\left\{\frac{\partial G}{\partial a} \frac{\partial \chi_{a p-b r}}{\partial b}-\frac{\partial G}{\partial b} \frac{\partial \chi_{a p-b r}}{\partial a}\right\}
$$

so folgt

$$
\mathbf{G G R} \chi_{a P_{f}-b R_{f}}=\frac{1}{4} \gamma \chi_{a p-b r}\left\{\frac{\partial G}{\partial \dot{a}} \Delta f-\frac{\partial G}{\partial b} f\right\}-\frac{1}{4}(a f+b \Delta f)\left\{\frac{\partial \chi_{a p-b r}}{\partial b} \frac{\partial G}{\partial a}-\frac{\partial \chi_{a p-b r}}{\partial a} \frac{\partial G}{\partial b}\right),
$$

welche Gleichung mit Berücksichtigung von

$$
\gamma \cdot \chi_{a p-b r}=\frac{\partial \chi_{a p-b r}}{\partial a} a+\frac{\partial \chi_{a p-b r}}{\partial b} b
$$

sofort in die zu beweisende Formel (8.) übergeht.

Die hier entwickelten Theoreme sind äusserst zahlreicher Anwendungen fähig; beispielsweise ergeben sich aus ihnen die meisten von Herrn Aronhold auf Seite 191 seiner Abhandlung aufgestellten Formeln fast ohne alle Rechnung.

Das Interesse für diese Sätze wird noch durch den Umstand erhöht, dass sie sämmtlich ihre Analoga in der Theorie der binären cubischen und biquadratischen Formen haben, wie man in meiner Habilitationsschrift näher sehen kann.

Tübingen, im October 1870. 